\title{
Growth and Continuity of Functions Satisfying Quadratic Integral Inequalities
}

\section{CHARLES A. GREENHALL}

\author{
Communicated by M. RosenblatT
}

1. Introduction. Let $f$ be a real measurable function on $[0,1]$. We are concerned with conditions on $f$ of the form

$$
I_{p}(f)=I(f)=\int_{0}^{1} \int_{0}^{1}\left[\frac{f(x)-f(y)}{p(x-y)}\right]^{2} d x d y<\infty .
$$

Here, $p$ is a continuous function on $[-1,1]$ such that

$$
p(x)=p(-x)>0 \text { for } x>0, \quad p \uparrow \text { on }[0,1], p(0)=0 .
$$

A. Garsia, E. Rodemich, and H. Rumsey [3, 4] considered a more general condition

$$
I_{\Psi, p}(f)=\int_{0}^{1} \int_{0}^{1} \Psi\left(\frac{f(x)-f(y)}{p(x-y)}\right) d x d y<\infty,
$$

where $\Psi$ is a function on $(-\infty, \infty)$ satisfying

$$
\Psi(x)=\Psi(-x), \Psi \uparrow \text { on }[0, \infty), \lim _{\infty \rightarrow x} \Psi(x)=\infty .
$$

Their theorem is

Theorem 1.1. Assume that

$$
\begin{gathered}
I_{\Psi, p}(f) \leqq B, \\
\int_{0}^{1} \Psi^{-1}\left(\frac{B}{t^{2}}\right) d p(t)<\infty .
\end{gathered}
$$

Then we may make $f$ continuous by modifying it on a set of measure 0 . The modified $f$ satisfies

$$
|f(x)-f(y)| \leqq 8 \int_{0}^{|x-y|} \Psi^{-1}\left(\frac{4 B}{t^{2}}\right) d p(t)
$$

for all $x, y$ in $[0,1]$. 
If the integral in (1.6) is infinite then the theorem says nothing. In this case, however, there is another theorem [2], which estimates the rate of growth of $f$ :

Theorem 1.2. Suppose $f$ is decreasing on $(0,1)$ and satisfies (1.5). Then

$$
f(x)-f\left(\frac{1}{2}\right) \leqq 4 \Psi^{-1}\left(\frac{B}{x^{2}}\right) p(x)+4 \int_{x}^{1 / 2} \Psi^{-1}\left(\frac{B}{t^{2}}\right) d p(t)
$$

for $0<x \leqq \frac{1}{2}$.

Obviously the same estimate holds for $f\left(\frac{1}{2}\right)-f(1-x)$.

The proofs of these theorems are easy, but the extension of Theorem 1.2 to non-monotonic functions requires a more difficult theorem of Garsia, Rodemich, and H. Taylor $[2,8]$. Let $f^{*}$ be a decreasing rearrangement of $f$, i.e., $f^{*} \downarrow$ on $(0,1)$ and the sets $\{f>\lambda\},\left\{f^{*}>\lambda\right\}$ have equal measure, for all $\lambda$.

Theorem 1.3. Let $\Psi$ and $p$ satisfy (1.4) and (1.2). Assume also that $\Psi\left(e^{x}\right)$ is convex. Then

$$
I_{\Psi, p}\left(f^{*}\right) \leqq I_{\Psi, p}(f) .
$$

Therefore, provided $\Psi\left(e^{x}\right)$ is convex, we can apply Theorem 1.2 to $f^{*}$ and obtain: If $f$ is measurable and $I(f) \leqq B$, then (1.8) holds with $f$ replaced by $f^{*}$.

The results in the present paper are also of this nature-we prove them for decreasing $f$ and use Theorem 1.3 to extend them to general $f$. Our ultimate aim is to get best possible versions of Theorems 1.1 and 1.2 for as wide a variety of $\Psi$ and $p$ as possible. For the present, we develop a theory for $\Psi(x)=x^{2}$ and obtain concrete results for $p(x)=x^{\alpha}$ or $x \log ^{\beta}(1 / x)$.

Some conventions are in order. First, when we say $p(x)=x^{\alpha} \log ^{\beta}(1 / x)$, we mean this to hold only on some unspecified interval $(0, \delta]$. Secondly, when we say that $\theta(x)$ is like $\varphi(x)$ for $x$ in some domain, we mean that $\theta(x) / \varphi(x)$ is bounded away from 0 and $\infty$. The bounds may or may not be uniform in others parameters involved. Thirdly, $\theta(x) \sim \varphi(x)$ as $x \rightarrow 0$ means $\theta(x) / \varphi(x) \rightarrow 1$ as $x \rightarrow 0$.

From now on, $\Psi(x)=x^{2}$, and $p$ satisfies (1.2). If $0<a<b<1$ define

$$
\sigma[a, b]=\sup \left\{f(a)-f(b): f \downarrow \text { on }(0,1), I_{p}(f) \leqq 1\right\} .
$$

Clearly, we may restrict ourselves to those $f$ which are constant on $[0, a]$ and $[b, 1]$. Here is a typical "growth result" of this paper: Let $p(x)=x$. Then $\sigma\left[x, \frac{1}{2}\right]$ is like $\log ^{1 / 2}(1 / x)$ for $0<x<1 / 4$. From Theorem 1.3 it follows that

$$
I(f) \leqq 1 \Rightarrow f^{*}(x)-f^{*}\left(\frac{1}{2}\right) \leqq \text { const } \log ^{1 / 2}(1 / x), \quad 0<x<1 / 4 .
$$

Furthermore, this result is best possible. On the other hand, Theorem 1.2 plus Theorem 1.3 gives only

$$
I(f) \leqq 1 \Rightarrow f^{*}(x)-f^{*}\left(\frac{1}{2}\right) \leqq 4-4 \log 2+4 \log (1 / x) .
$$

In this connection we should mention the well-known theorem of John and 
Nirenberg [5] on functions of "bounded mean oscillation." A function $f \varepsilon L^{1}(0,1)$ is said to be of B.M.O. if there is a constant $M$ such that

$$
\frac{1}{|I|} \int_{I}\left|f-f_{I}\right| \leqq M
$$

for all subintervals $I$ of $[0,1]$. Here, $|I|$ means the length of $I$, and

$$
f_{I}=\frac{1}{|I|} \int_{I} f
$$

The John-Nirenberg Theorem gives that if $f$ is of B.M.O. then

$$
f^{*}(x)-\int_{0}^{1} f \leqq \text { const }+ \text { const } \log (1 / x),
$$

which is essentially (1.11). It is easy to see, however, that

$$
\frac{1}{|I|} \int_{I}\left|f-f_{I}\right| \leqq \int_{0}^{1} \int_{0}^{1}\left[\frac{f(x)-f(y)}{x-y}\right]^{2} d x d y
$$

for all $I \subset[0,1]$. In other words, $I(f)<\infty$ is stronger than the B.M.O. condition, and (1.10) is an appropriately stronger result.

For $p(x)=x^{1-\epsilon}, 0<\epsilon<\frac{1}{2}$, the growth result from Theorems 1.2 and 1.3 agrees with the best-possible result our method attains, namely, $\sigma\left[x, \frac{1}{2}\right]$ is like $x^{-\epsilon}$. For $p(x)=x^{1+\epsilon}$, both methods predict that any $f$ with $I(f)<\infty$ is essentially continuous, with modulus of continuity $\leqq$ const $x^{\epsilon}$. For $p(x)=x$, we saw that the older result from Theorem 1.2 is too big by a factor $\log ^{1 / 2}(1 / x)$. If we then look at the "boundary cases" $p(x)=x \log ^{\beta}(1 / x)$, we find a range of $\beta$ such that $I(f)<\infty$ implies $f$ essentially continuous, yet the integral in (1.6) is infinite. Hence the older results do not predict continuity, but only a certain rate of growth. If $\beta=-1$, for example, our modulus-of-continuity estimate is like $\log ^{-1 / 2}(1 / x)$, whereas Theorem 1.2 gives a rate of growth $\leqq$ const $\log \log$ $(1 / x)$.

2. Application of potential theory. Our method uses the theory of capacity. The set of $f$ satisfying (1.1) is a Dirichlet space in the sense of Beurling and Deny. But the capacities we use are not with respect to this space. These $f$ do not play the role of potentials. Indeed, we will transform $I(f)$ in such a way that $d f$ takes the role of a charge distribution whose energy is $I(f)$.

Define the positive symmetric kernel $K$ by

$$
K(x, y)=K(y, x)=2 \int_{0}^{x} d s \int_{y}^{1} d t \frac{1}{p^{2}(t-s)}, \quad 0 \leqq x \leqq y \leqq 1 .
$$

Obviously $K(x, 0)=K(0, x)=0$, and $K(1-x, 1-y)=K(x, y)$.

Theorem 2.1. For any monotonic function $f$ on $(0,1)$, 


$$
I(f)=\int_{0}^{1} \int_{0}^{1} K(x, y) d f(x) d f(y) .
$$

Proof. We first write

$$
I(f)=2 \int_{0}^{1} d x \int_{x}^{1} d y\left[\frac{f(y)-f(x)}{p(y-x)}\right]^{2} .
$$

We may assume that $f$ is right-continuous, and replace $[f(y)-f(x)]^{2}$ by

$$
\int_{(x, y]} d f(s) \int_{(x, y]} d f(t), \quad 0<x<y<1 .
$$

Then interchanging orders of integration gives (2.2). Observe that jumps of $f$ at the endpoints affect neither side of (2.2).

If $\mu$ and $\nu$ are positive Borel measures on $(0,1)$ we write

$$
\begin{aligned}
E(\mu, \nu) & =\int_{0}^{1} \int_{0}^{1} K(x, y) d \mu(x) d \nu(y), \\
E(\mu) & =E(\mu, \mu),
\end{aligned}
$$

and also use this notation for signed measures $\mu$ and $\nu$, provided $E(|\mu|)$ and $E(|\nu|)$ are finite.

Corollary 2.1. i) If $f$ is of locally bounded variation on $(0,1)$ with total variation function $v$ and $I(v)<\infty$, then $I(f)<\infty$ and (2.2) holds.

ii) The kernel $K$ is positive definite in the sense that for any signed measure $\mu$,

$$
\mu \neq 0, E(|\mu|)<\infty \Rightarrow E(\mu)>0 .
$$

Proof. (i) follows from $|f(x)-f(y)| \leqq|v(x)-v(y)|$ and the Jordan decomposition.

To prove (ii), let $\mu=d f$. If $E(|\mu|)<\infty$ and $E(\mu)=0$, then $I(f)=0$, by (i). Hence $f(x)-f(y)=0$ for a.e. $(x, y)$. Hence $\mu=0$.

We now interpret a measure $\mu$ on $(0,1)$ as a charge distribution, and $K(x, \cdot)$ as the potential of a unit charge at $x$ between two grounded points 0 and 1 . The function $u_{\mu}$ defined by

$$
u_{\mu}(x)=\int_{0}^{1} K(x, y) d \mu(y)
$$

is the potential of $\mu$; the energy of $\mu$ is

$$
E(\mu)=\int_{0}^{1} u_{\mu} d \mu
$$

the mutual energy of $\mu$ and $\nu$ is

$$
E(\mu, \nu)=\int_{0}^{1} u_{\mu} d \nu=\int_{0}^{1} u_{\nu} d \mu .
$$


Let $S$ be a subset of $(0,1)$ and set

$$
\sigma(S)=\sup \mu(0,1),
$$

the sup being over all positive measures $\mu$ with closed support in $S$ such that $E(\mu) \leqq 1$. In other words, $\sigma(S)$ is the most charge you can put on $S$ while keeping the energy $\leqq 1$. For $S=[a, b]$ this is the same as $\sigma[a, b]$ defined by (1.9).

The set function $\sigma$ is directly related to another set function $C$. The capacity $C(S)$ of any $S \subset(0,1)$ is defined as

$$
C(S)=\sup \mu(0,1),
$$

the sup being over positive measures $\mu$ with closed support in $S$ such that $u_{\mu} \leqq 1$ on $S$. Thus $C(S)$ is the most charge you can put in $S$ while keeping the potential $\leqq 1$. The theory of capacity can be found in L. Carleson's book [1] and C. Preston's thesis [6]. The essential fact is that, for compact $F$, the variational problem $\sigma(F)$ has a unique solution $\mu$ which is the solution of the integral equation

$$
u_{\mu}(x)=\int_{F} K(x, y) d \mu(y)=\text { const }
$$

for all $x \varepsilon F$ minus a set of capacity 0 .

Since Carleson and Preston present the theory for translation kernels $K(x-y)$, we will run through the sequence of theorems needed for our work. The proofs that can be found in [1] or [6] will be omitted but we will point out the properties of our $K$ that make the proofs go through.

Theorem 2.2. Let $\left(\mu_{n}\right)$ be a sequence of positive finite measures on $[0,1]$ which converges weakly to $\mu$. Then

$$
\lim _{n \rightarrow \infty} \inf E\left(\mu_{n}\right) \geqq E(\mu) .
$$

For Preston's proof we merely need to have a sequence of continuous functions $K_{m}$ that increases to $K$. For example, we may set

$$
p_{m}(x)=\max (1 / m, p(x))
$$

and define $K_{m}$ by (2.1), with $p$ replaced by $p_{m}$.

Lemma 2.1. For each $x \varepsilon(0,1)$ the function $K(x, \cdot)$ is convex on the intervals $[0, x)$ and $(x, 1]$ (on $[0, x]$ and $[x, 1]$ if $K(x, \cdot)$ is continuous).

Proof.

$$
\begin{aligned}
\frac{\partial^{2}}{\partial y^{2}} K(x, y) & =\frac{2}{p^{2}(y-x)}-\frac{2}{p^{2}(y)}, \quad y>x \\
& =\frac{2}{p^{2}(x-y)}-\frac{2}{p^{2}(1-y)}, \quad y<x .
\end{aligned}
$$

Since $p$ is increasing on $[0,1]$, the second derivative of $K(x, \cdot)$ is nonnegative. 
Theorem 2.3. (The Maximum Principle) If $\mu$ is a positive measure on $(0,1)$ and $u_{\mu} \leqq 1$ on the closed support $S_{\mu}$ of $\mu$, then $u_{\mu} \leqq 1$ everywhere.

Proof. Our situation resembles Carleson's, rather than Preston's. As we are working in one dimension, the proof is easy, and we will give it.

Let $I$ be a component interval of $(0,1) \backslash S_{\mu}$. Then $u_{\mu} \leqq 1$ at the endpoints of $I$. Hence we will be done if we show $u_{\mu}$ is convex on $\bar{I}$. But this is an easy consequence of the convexity of $K$, as follows: If $x, y \varepsilon \bar{I}, 0 \leqq \lambda \leqq 1$, $z=(1-\lambda) x+\lambda y, t \notin I$, then by Lemma 2.1 ,

$$
K(z, t) \leqq(1-\lambda) K(x, t)+\lambda K(y, t) .
$$

(One or both sides may be $\infty$ ). Now integrate this with respect to $d \mu(t)$ over ${ }^{c} I$ to obtain

$$
u_{\mu}(z) \leqq(1-\lambda) u_{\mu}(x)+\lambda u_{\mu}(y) .
$$

Theorem 2.4. The set function $C$ is increasing and countably subadditive.

Theorem 2.5. Let $S \subset(0,1)$ be a Borel set and $\mu$ a positive measure. If $u_{\mu}$ is bounded on $S$ and $C(S)=0$ then $\mu(S)=0$.

The proof of the main theorem to come involves the set function

$$
\tau(S)=\inf E(\mu)^{1 / 2}
$$

the inf being over measures $\mu$ supported in $S$ such that $\mu(S) \geqq 1$. The functions $\tau$ and $\sigma$ are obviously reciprocal:

Lemma 2.2. $\sigma(S)=1 / \tau(S)$ (where $1 / \infty=0,1 / 0=\infty)$.

Proof. Assume $0<\tau(S)<\infty, 0<\sigma(S)<\infty$. Suppose $\mu$ is supported in $S, E(\mu) \leqq 1$. Then

$$
E(\theta \tau(S) \mu)<\tau^{2}(S)
$$

for all $0<\theta<1$. By the definition of $\tau(S), \theta \tau(S) \mu(S)<1$ and hence $\mu(S)<$ $1 / \theta \tau(S)$ for all $0<\theta<1$. This shows $\sigma(S) \leqq 1 / \tau(S)$. By going the other way, we prove $\sigma(S) \geqq 1 / \tau(S)$.

The cases $\tau(S)$ or $\sigma(S)=0, \infty$ are just as easily handled.

If something happens except on a set of capacity 0 , we say it happens $C$-a.e.

Here is the main result.

Theorem 2.6. Let $F$ be a compact subset of $(0,1)$. Then $C(F)<\infty$, and $C(F)=\sigma^{2}(F)$. There is a unique measure $\mu_{0}$ supported in $F$, called the equilibrium measure for $F$, such that

i) $u_{\mu_{0}} \leqq 1$ everywhere, $u_{\mu_{0}}=1$-a.e. on $F$

ii) $\mu_{0}(F)=C(F)$.

Furthermore, the $\sigma(F)$ and $\tau(F)$ variational problems are both solved uniquely by multiples of $\mu_{0}$, namely $\mu_{0} / \sigma(F)$ and $\mu_{0} / C(F)$. 
In fact, the solution $\mu_{1}$ of the $\tau(F)$ problem is first obtained as a weak limit of approximate solutions. It is proved that $u_{\mu_{1}}=$ const $C$-a.e. on $F$. Then $\mu_{0}$ is defined as a multiple of $\mu_{1}$.

We are finally at the point of application to our own problem. Recall that we wanted to evaluate

$$
\begin{aligned}
\sigma[a, b] & =\sup \{f(a)-f(b): f \downarrow, \quad I(f) \leqq 1\} \\
& =\sup \{\mu[a, b]: \mu \text { positive, } E(\mu) \leqq 1\} .
\end{aligned}
$$

Letting $[a, b]=F$ in Theorem 2.6, we see that there is a maximizing $f$ for this problem, unique to within an additive constant and values at jumps. To obtain $\sigma[a, b]$ we may solve the integral equation

$$
\begin{aligned}
\int_{[a, b]} K(x, y) d \mu_{0}(y) & \leqq 1 \text { on } F \\
& =1 \quad C \text {-a.e. on } F
\end{aligned}
$$

for the positive measure $\mu_{0}$. Then $C[a, b]=\mu_{0}[a, b]$ and $\sigma[a, b]=C[a, b]^{1 / 2}$.

We do not, however, demand to calculate $\sigma[a, b]$ exactly. We will be content here to estimate $\sigma[a, b]$ to within multiplicative constants for fixed $b$ and all small $a$. To do this we do not have to solve (2.3) exactly; an approximate solution will do. More precisely,

Theorem 2.7. Suppose the measure $\mu$ is supported in the campact set $F$, and there are positive constants $m, M$ such that

$$
m \leqq u_{\mu} \leqq M \text { on } F \text {. }
$$

Then

$$
\mu(F) / M \leqq C(F) \leqq \mu(F) / m .
$$

Proof. Let $\mu_{0}$ be the equilibrium measure for $F$. Then

$$
E\left(\mu, \mu_{0}\right)=\int_{F} u_{\mu_{0}} d \mu=\int_{F} u_{\mu} d \mu_{0} .
$$

We know $u_{\mu_{0}}=1$ on $F$ except for a set of capacity 0 . Since $\mu$ has a bounded potential, this set has $\mu$-measure 0 , by Theorem 2.5. Therefore

$$
\int_{F} u_{\mu_{0}} d \mu=\mu(F)
$$

On the other hand, $\mu_{0}(F)=C(F)$ and

$$
m \mu_{0}\left(F^{\prime}\right) \leqq \int_{F} u_{\mu} d \mu_{0} \leqq M \mu_{0}\left(F^{\prime}\right)
$$

3. The approximate equilibrium measures. Given $p$ satisfying (1.2), we are trying to calculate a function $\varphi(x)$ such that $\sigma[x, b]$ (for some fixed $b$ ) is like $\varphi(x)$ for all small $x$. According to Theorem 2.7, we can do this by finding, for 
each $x$, a measure $\mu_{x}$ which sits on $[x, b]$ and whose potential function is like 1 on $[x, b]$, uniformly in $x$. Then $\sigma^{2}[x, b]$ is like $\mu_{x}[x, b]$. We have been able to do this for special cases of $p$ by the following heuristic argument. Suppose that $K(x, y)$ is like a $\delta$-function: $K(x, y)=k(x) \delta(y-x)$, and we look for a measure $\rho(y) d y$ whose potential is 1 . Thus

$$
\begin{aligned}
\int K(x, y) \rho(y) d y & =k(x) \rho(x)=1, \\
\rho(x) & =1 / k(x) .
\end{aligned}
$$

Now we might identify $k(x)$ with $\int_{0}^{1} K(x, y) d y$. If $x$ is near 0 , however, the long tail of $K(x, \cdot)$ extending toward 1 contributes too much to this integral. Therefore we use $\int_{0}^{x} K(x, y) d y$ for $k(x)$, and set

$$
\rho(x)=\left[\int_{0}^{x} K(x, y) d y\right]^{-1} .
$$

We must then look at the potential of the measure $\rho(y) d y$, restricted to $[x, b]$.

It will be useful to have an asymptotic approximation for $K(x, y), x$ and $y$ small. Define

$$
P(x)=\int_{x}^{1} \frac{d u}{p^{2}(u)}, \quad 0<x \leqq 1 .
$$

If $P \varepsilon L^{1}(0,1)$ let

$$
q(x)=2 \int_{0}^{x} P(t) d t, \quad 0 \leqq x \leqq 1
$$

If $P \& L^{1}(0,1)$ let

$$
q(x)=2 \int_{x}^{1} P(t) d t, \quad 0<x \leqq 1 .
$$

Theorem 3.1.

$$
K(x, y) \sim|q(y)-q(y-x)|, \quad x<y, \quad x \rightarrow 0,
$$

uniformly in $y$ for $0<y \leqq \frac{1}{2}$.

Proof. For $x<y$,

$$
\begin{aligned}
K(x, y) & =2 \int_{0}^{x} d s \int_{y-s}^{1-s} \frac{d u}{p^{2}(u)} \\
& =2 \int_{0}^{x}[P(y-s)-P(1-s)] d s .
\end{aligned}
$$

If $0 \leqq s \leqq x<y \leqq \frac{1}{2}, x \leqq \delta$, then

$$
\frac{P(1-s)}{P(y-s)} \leqq \frac{P(1-\delta)}{P\left(\frac{1}{2}\right)},
$$


which tends to 0 with $\delta$. Therefore as $x \rightarrow 0$,

$$
K(x, y) \sim 2 \int_{0}^{x} P(y-s) d s=2 \int_{y-x}^{y} P(t) d t,
$$

and the theorem follows.

We will actually use (3.4) rather than (3.3).

\section{Corollary 3.1.}

$$
\int_{0}^{x} K(x, y) d y \sim 2 \int_{0}^{x} P(t) t d t \quad \text { as } \quad x \rightarrow 0 .
$$

Proof. We just plug in the right side of (3.4) for $K(x, y)$ (with $x$ and $y$ interchanged), and interchange orders of integration.

It is possible that the integral

$$
\int_{0}^{1} P(t) t d t=\int_{0}^{1} \frac{t^{2} d t}{p^{2}(t)}
$$

is infinite. Later, we will show that in this case the only functions $f$ with $I(f)<\infty$ are the constants.

4. Capacities for $p(x)=x^{\alpha}, \frac{1}{2}<\alpha<\frac{3}{2}$. Growth results when $\frac{1}{2}<\alpha \leq 1$. For these $p$ we get, from (3.1), (3.2), and (3.5), that

$$
\begin{aligned}
& P(x) \sim \frac{1}{(2 \alpha-1)^{2 \alpha-1}}, \\
& \rho(x) \sim \frac{(2 \alpha-1)(3-2 \alpha)}{2} \frac{1}{x^{3-2 \alpha}} \text { as } x \rightarrow 0 .
\end{aligned}
$$

We will reset

$$
\rho(x)=\frac{1}{x^{3-2 \alpha}}
$$

and test the potential $u$ of the measure $\rho(y) d y$ on intervals $[a, b]$ for likeness to 1 on $[a, b]$, uniformly in $a$ and $b$.

First define

$$
\begin{aligned}
v(s, t) & =\int_{s}^{t} K(t, y) \rho(y) d y \\
w(s, t) & =\int_{s}^{t} K(s, y) \rho(y) d y .
\end{aligned}
$$

Then

$$
u(x)=v(a, x)+w(x, b), \quad a \leqq x \leqq b .
$$


Lemma 4.1. If $0<2 s \leqq t$ and $t$ is small enough, then $v(s, t)$ and $w(s, t)$ are both like 1 .

Proof. If we keep $t$ small enough, then whenever $K$ occurs we may use (3.4).

Take $v$ first. Write

$$
v(s, t)=\int_{t / 2}^{t} K(t, y) \rho(y) d y+\int_{s}^{t / 2} K(t, y) \rho(y) d y=v_{1}+v_{2} .
$$

For $t / 2 \leqq y \leqq t, \rho(y)$ is like $\rho(t)$, for all the $\rho$ under consideration. Accordingly, $v_{1}^{\prime}$ is like

$$
\rho(t) \int_{t / 2}^{t} d y \int_{t-y}^{t} d u P(u)=\rho(t)\left[t / 2 \int_{t / 2}^{t} P(u) d u+\int_{0}^{t / 2} P(u) u d u\right] .
$$

This is like 1, as can immediately be verified for the various $\alpha$.

The quantity $v_{2}$ is like

$$
\int_{0}^{t / 2} d y \rho(y) \int_{t-y}^{t} d u P(u)
$$

The inner integral is like $y P(t)$ since $P(t / 2)$ is like $P(t)$. Hence

$$
v_{2} \leqq \text { const } P(t) \int_{0}^{t / 2} \rho(y) y d y \leqq \text { const. }
$$

Therefore $v=v_{1}+v_{2}$ is like 1 .

We similarly write

$$
w(s, t)=\int_{s}^{2 s} K(s, y) \rho(y) d y+\int_{2 s}^{t} K(s, y) \rho(y) d y=w_{1}+w_{2}
$$

First, $w_{1}$ is like

$$
\rho(s) \int_{s}^{2 s} d y \int_{y-s}^{y} d u P(u)=\rho(s) \int_{s}^{2 s} d y \int_{s}^{y} d u P(u)+\rho(s) \int_{s}^{2 s} d y \int_{y-s}^{s} d u P(u) .
$$

The first term of the right side is like

$$
\rho(s) P(s) \int_{0}^{2 s}(y-s) d y
$$

and the second term is precisely

$$
\rho(s) \int_{0}^{s} P(u) u d u .
$$

Both of these are like 1.

The term $w_{2}$ is like

$$
\int_{2 s}^{t} d y \rho(y) \int_{y-s}^{y} d u P(u)
$$


The inner integral is like $s P(y)$. Therefore

$$
w_{2} \leqq \text { const } s \int_{s}^{t} \rho(y) P(y) d y \leqq \text { const } s \int_{s}^{\infty} y^{-2} d y=\text { const. }
$$

This proves that $w$ is like 1 .

Theorem 4.1. Let $p(x)=x^{\alpha}, \frac{1}{2}<\alpha<\frac{3}{2}$. For $0<a \leqq b / 4$, and $b$ sufficiently small, the potential $u$ of the measure $y^{2 \alpha-3} d y$ on $[a, b]$ is like 1 on $[a, b]$, uniformly in $a$ and $b$ (but not necessarily in $\alpha$ ).

Proof. Observe that $v(s, t)$ is a decreasing function of $s$, and $w(s, t)$ is an increasing function of $t$.

Suppose first that $a \leqq x \leqq 2 a$. Then $v(a, x) \leqq v(x / 2, x)$, and so by (4.1),

$$
w(x, b) \leqq u(x) \leqq v(x / 2, x)+w(x, b) .
$$

By Lemma 4.1, the left and right sides of this are both like 1 if $b$ is small enough, since $2 x \leqq b$.

Suppose next that $2 a \leqq x \leqq b / 2$. Then both $v(a, x)$ and $w(x, b)$ are like 1 .

Finally, if $b / 2 \leqq x \leqq b$, then $w(x, b) \leqq w(x, 2 x)$, and so

$$
v(a, x) \leqq u(x) \leqq v(a, x)+w(x, 2 x) .
$$

The left and right sides are like 1 , since $2 a \leqq x$.

According to Theorem 2.7, $C[a, b]$ is now like

$$
\int_{a}^{b} y^{2 \alpha-3} d y
$$

and by Theorem 2.6, $\sigma[a, b]=C[a, b]^{1 / 2}$. Therefore we get:

Theorem 4.2. Let $p(x)=x^{\alpha}, \frac{1}{2}<\alpha<\frac{3}{2}$. For $0<a \leqq b / 4$ and $b$ sufficiently small, $\sigma[a, b]$ is like

$$
\begin{aligned}
& a^{\alpha-1} \quad \text { if } \quad \frac{1}{2}<\alpha<1 \\
& \log ^{1 / 2}(b / a) \text { if } \alpha=1 \\
& b^{\alpha-1} \quad \text { if } 1<\alpha<\frac{3}{2}
\end{aligned}
$$

(nonuniformly in $\alpha$ ).

From this, we obtain growth results in the first two cases, where $\sigma[a, b] \rightarrow \infty$ as $a \rightarrow 0$.

Theorem 4.3. Let $p(x)=x^{\alpha}, \frac{1}{2}<\alpha \leqq 1$. For sufficiently small $x, \sigma\left[x, \frac{1}{2}\right]$ is like

$$
\begin{aligned}
x^{\alpha-1} & \text { if } \quad \frac{1}{2}<\alpha<1 \\
\log ^{1 / 2}(1 / x) & \text { if } \quad \alpha=1
\end{aligned}
$$


Proof. The set function $\sigma$ is obviously monotone and subadditive. If $0<x<b \leqq \frac{1}{2}$ then

$$
\sigma[x, b] \leqq \sigma\left[x, \frac{1}{2}\right] \leqq \sigma[x, b]+\sigma\left[b, \frac{1}{2}\right] .
$$

We fix $b$ small enough for Theorem 4.2. Then $\sigma\left[x, \frac{1}{2}\right] \sim \sigma[x, b]$ as $x \rightarrow 0$. Theorem 4.2 completes the proof.

For $\frac{1}{2}<\alpha<1$, this growth result is like the Theorem 1.2 estimate, but for $\alpha=1$, Theorem 1.2 gives only $\log (1 / x)$.

5. Continuity results for $p(x)=x^{\alpha}, 1<\alpha<\frac{3}{2}$. For this case, Theorem 4.2 shows that for a fixed $b, \sigma[a, b]$ is bounded as $a \rightarrow 0$. This will quickly give that $I(f)<\infty$ implies $f$ bounded. Define

$$
\sigma(0, b]=\sup \{f(0+)-f(b): f \downarrow, \quad I(f) \leqq 1\} .
$$

Lemma 5.1. For each $b$ in $(0,1)$,

$$
\sigma(0, b]=\lim _{x \rightarrow 0} \sigma[x, b] .
$$

Proof. The limit exists since $\sigma[x, b] \uparrow$ as $x \downarrow$.

It is obvious that " $\geqq$ " holds, for if $I(f) \leqq 1, f \downarrow$, then

$$
f(x)-f(b) \leqq f(0+)-f(b) \leqq \sigma(0, b] .
$$

Let $L$ be the above limit, and suppose $\sigma(0, b]>L$. Then there is an $f \downarrow$ with $I(f) \leqq 1$ and $f(0+)-f(b)>L$. But then for small $x, f(x)-f(b)>L$. Hence $\sigma[x, b]>L$, which is impossible.

Theorem 5.1. Let $p(x)=x^{\alpha}, 1<\alpha<\frac{3}{2}$.

i) For small $x, \sigma(0, x]$ is like $x^{\alpha-1}$.

ii) If $I(f)<\infty$, then $f$ is essentially bounded.

Proof. (i) follows from Lemma 5.1 and Theorem 4.2. For (ii) we observe

$$
\sigma\left(0, \frac{1}{2}\right] \leqq \sigma(0, x]+\sigma\left[x, \frac{1}{2}\right], \quad 0<x<\frac{1}{2} .
$$

Therefore $\sigma\left(0, \frac{1}{2}\right]$ is finite. By symmetry, so is the corresponding quantity $\sigma\left[\frac{1}{2}, 1\right)$. Therefore we have (ii) for decreasing $f$. But then Theorem 1.3 gives it for general $f$, since $f \varepsilon L^{\infty}$ if and only if $f^{*}$ is bounded.

As we pointed out in the introduction, Theorem 1.1 predicts continuity for $f$ if $I(f)<\infty$ and $p(x)=x^{\alpha}, \alpha>1$. Indeed, (1.7) gives

$$
|f(x)-f(y)| \leqq \frac{16 \alpha}{\alpha-1}|x-y|^{\alpha-1}
$$

if $I(f) \leqq 1$. Theorem 5.1 (i) gives a like modulus of continuity for monotone functions at the endpoints. We now set out to show that we can make our method predict not only boundedness, but continuity, of any $f$ with $I(f)<\infty$.

We use the following change of scale argument, which was also used in one 
proof of Theorem 1.1. Return to a general $p$ satisfying (1.2). For $0<r \leqq 1$ let $I_{r}(f)$ denote the integral (1.1), except that $p(x)$ is replaced by the function $p_{r}(x)=p(r x)$.

Suppose $I(f) \leqq 1$. Then for $0 \leqq x<y \leqq 1$,

$$
\int_{x}^{y} \int_{x}^{y}\left[\frac{f(s)-f(t)}{p(s-t)}\right]^{2} d s d t \leqq 1 .
$$

Let $r=y-x$. If we make the change of variables $s=x+r u, t=y+r v$, then (5.1) becomes

$$
I_{r}(g) \leqq 1 / r^{2}
$$

where $g(u)=f(x+r u), 0 \leqq u \leqq 1$.

Define $\sigma_{r}(S)$ using the integral $I_{r}$, just as $\sigma(S)$ is defined using $I$. In particular let

$$
\sigma_{r}(0,1)=\sup \left\{g(0+)-g(1-): g \downarrow, I_{r}(g) \leqq 1\right\} .
$$

Then (5.2) and Theorem 1.3 imply

$$
I_{r}\left(r g^{*}\right) \leqq 1
$$

and hence

$$
g^{*}(0+)-g^{*}(1-) \leqq r^{-1} \sigma_{r}(0,1) .
$$

Translating back to $f$ we have

Lemma 5.2. Let $I(f) \leqq 1$ (general $p$ ). If $0 \leqq x<y \leqq 1$, and $r=y-x$, then for a.e. $s$ and a.e. $t$ in $[x, y]$,

$$
|f(s)-f(t)| \leqq r^{-1} \sigma_{r}(0,1) .
$$

To investigate $\sigma_{r}(0,1)$, we look at the kernel $K_{r}$ associated with the integral $I_{r}$. For $x<y$,

$$
K_{r}(x, y)=2 \int_{0}^{x} d s \int_{\nu}^{1} d t \frac{1}{p^{2}(r(t-s))}
$$

Recall the definition of $P$ in (3.2).

Lemma 5.3. Assume that $P(2 x) / P(x)$ is bounded away from 1 for $0<x \leqq \frac{1}{2}$. Then $K_{r}(x, y)$ is like

$$
r^{-2} K(r x, r y)
$$

for $0 \leqq x, y \leqq \frac{1}{2}, 0<r \leqq 1$.

Proof. For $x<y$,

$$
K_{r}(x, y)=\frac{2}{r^{2}} \int_{0}^{r x} d s \int_{r y}^{r} d t \frac{1}{p^{2}(t-s)}
$$




$$
=\frac{2}{r^{2}} \int_{0}^{r x}[P(r y-s)-P(r-s)] d s .
$$

On the other hand, from the proof of Theorem 3.1,

$$
\frac{1}{r^{2}} K(r x, r y)=\frac{2}{r^{2}} \int_{0}^{r x}[P(r y-s)-P(1-s)] d s .
$$

Consider (5.4). If $0 \leqq s \leqq r x<r y \leqq r / 2$, then

$$
(r-s) /(r y-s) \geqq r /(r y) \geqq 2 .
$$

Therefore, by our assumption, $P(r-s) / P(r y-s)$ is bounded away from 1 (from below). This is also true of the ratio $P(1-s) / P(r y-s)$ from (5.5), since $r-s \leqq 1-s$. It follows that both $K_{r}(x, y)$ and $r^{-2} K(r x, r y)$ are like

$$
\frac{2}{r^{2}} \int_{0}^{r x} P(r y-s) d s
$$

uniformly in $r$.

By relating $K$ and $K_{r}$, we can also relate the corresponding capacities $C$ and $C_{r}$.

Lemma 5.4. For any set $S \subset\left(0, \frac{1}{2}\right], C_{r}(S)$ is like $r^{2} C(r S)$ for $0<r \leqq 1$, where $r S=\{r x: x \varepsilon S\}$.

Proof. From the last lemma, there is an $m>1$ such that

$$
\frac{1}{m} \frac{K(r x, r y)}{r^{2}}<K_{r}(x, y)<m \frac{K(r x, r y)}{r^{2}}
$$

for $0 \leqq x, y \leqq \frac{1}{2}, 0<r \leqq 1$.

By the definition of $C_{r}(S)$, there is a measure $d f$ supported in $S$ such that

$$
\begin{gathered}
\int_{S} K_{r}(x, y) d f(y) \leqq 1, \quad x \varepsilon S, \\
\int_{S} d f \geqq \frac{1}{2} C_{r}(S) .
\end{gathered}
$$

Therefore

$$
\begin{gathered}
\int_{S} r^{-2} K(r x, r y) d f(y) \leqq m, \\
\frac{1}{m r^{2}} \int_{r S} K(r x, s) d f(s / r) \leqq 1, \quad r x \varepsilon r S .
\end{gathered}
$$

Since $d f(s / r)$ is supported in $r S$, this gives that

$$
\frac{1}{m r^{2}} \int_{r S} d f(s / r) \leqq C(r S)
$$




$$
\frac{1}{m r^{2}} \int_{S} d f \leqq C(r S)
$$

Combining this with (5.6), we get

$$
C_{r}(S) \leqq 2 m r^{2} C(r S)
$$

Working the argument the other way gives an opposite inequality.

It is now easy to prove a continuity result.

Theorem 5.2. Let the assumption on $P$ of Lemma 5.3 hold, and assume that $\sigma(0, x] \rightarrow 0$ as $x \rightarrow 0$. If $I(f) \leqq 1$, then we may make $f$ continuous on $[0,1]$ by adjusting it on a set of measure 0 . For the adjusted $f$,

$$
|f(x)-f(y)| \leqq \text { const } \sigma\left(0, \frac{1}{2}|x-y|\right], \quad 0 \leqq x, y \leqq 1,
$$

where the constant depends only on the function $p$.

Proof. From Lemma 5.4 and Theorem 2.6 we get that $\sigma_{r}\left[a, \frac{1}{2}\right]$ is like $r \sigma[r a, r / 2]$ for $0<a<\frac{1}{2}, 0<r \leqq 1$. Letting $a \rightarrow 0$ we see that $\sigma_{r}\left(0, \frac{1}{2}\right]$ is like $r \sigma(0, r / 2]$. Since $\sigma_{r}\left(0, \frac{1}{2}\right] \leqq \sigma_{r}(0,1) \leqq 2 \sigma_{r}\left(0, \frac{1}{2}\right]$, the right side of (5.3) may be replaced by

$$
\text { const } \sigma(0, r / 2]=\text { const } \sigma\left(0, \frac{1}{2}|x-y|\right] \text {. }
$$

A little real-variable theory then gives the essential continuity of $f$, plus the estimate (5.7).

Returning to the case $p(x)=x^{\alpha}, 1<\alpha<3 / 2$, we see from Theorems 5.1 and 5.2 that the functions $f$ with $I(f) \leqq 1$ are essentially continuous, and

$$
|f(x)-f(y)| \leqq \text { const }|x-y|^{\alpha-1} \text {. }
$$

6. The boundary cases $p(x)=x \log ^{\beta}(1 / x)$. We look at these $p$ in order to compare our results more closely with Theorems 1.1 and 1.2. From (3.1), (3.2), and (3.5),

$$
\begin{gathered}
P(x) \sim(1 / x) \log ^{-2 \beta}(1 / x) \text { as } x \rightarrow 0 . \\
\rho(x) \sim(1 / 2 x) \log ^{2 \beta}(1 / x)
\end{gathered}
$$

The reader may easily verify that Lemma 4.1 goes through as before. We then get the following growth and continuity results:

$$
\begin{aligned}
& \text { If } \beta>-\frac{1}{2} \text {, then } \sigma\left[x, \frac{1}{2}\right] \text { is like }\left(\log \frac{1}{x}\right)^{\beta+\frac{1}{2}} . \\
& \text { If } \beta=-\frac{1}{2} \text {, then } \sigma\left[x, \frac{1}{2}\right] \text { is like }\left(\log \log \frac{1}{x}\right)^{\frac{1}{2}} \\
& \text { If } \beta<-\frac{1}{2} \text {, then } \sigma(0, x] \text { is like }\left(\log \frac{1}{x}\right)^{\beta+\frac{1}{2}} .
\end{aligned}
$$

We compare these with Theorems $1.1,1.2$, and 1.3 , which give, for $I(f) \leqq 1$ : 


$$
\begin{aligned}
& \text { If } \beta>-1, \text { then } f^{*}(x)-f^{*}\left(\frac{1}{2}\right) \leqq \text { const }\left(\log \frac{1}{x}\right)^{\beta+1} . \\
& \text { If } \beta=-1, \text { then } f^{*}(x)-f^{*}\left(\frac{1}{2}\right) \leqq \text { const } \log \log \frac{1}{x} . \\
& \text { If } \beta<-1, \text { then }|f(x)-f(y)| \leqq \text { const }\left(\log \frac{1}{|x-y|}\right)^{\beta+1} .
\end{aligned}
$$

In general, these earlier estimates are too large by a factor $\log ^{1 / 2}(1 / x)$. Moreover, if $-1 \leqq \beta<\frac{1}{2}$, then (6.3) and Theorem 5.2 predict continuity of $f$ if $I(f)<\infty$, whereas (6.4) and (6.5) only predict a certain rate of growth.

7. The trivial case $p(x)=x^{\alpha}, \alpha \geqq \frac{3}{2}$. We will show that in this case the only functions $f$ with $I(f)<\infty$ are the constants. In fact, this happens if and only if the integral

$$
\int_{0}^{1} \frac{t^{2} d t}{p^{2}(t)}
$$

is infinite.

Lemma 7.1. If (7.1) is finite, then $K(x, \cdot) \varepsilon L^{1}(0,1)$ for all $x \varepsilon(0,1)$. If (7.1) is infinite, then $K(x, \cdot) \varepsilon L^{1}$ for no $x \varepsilon(0,1)$.

Proof. First observe

$$
\begin{aligned}
\int_{0}^{1} K(x, y) d y & =\int_{0}^{x} K(x, y) d y+\int_{x}^{1} K(x, y) d y \\
& =\int_{0}^{x} K(x, y) d y+\int_{0}^{1-x} K(1-x, y) d y
\end{aligned}
$$

Then

$$
\begin{aligned}
\int_{0}^{x} K(x, y) d y & =2 \int_{0}^{x} d y \int_{0}^{y} d s \int_{x}^{1} d t \frac{1}{p^{2}(t-s)} \\
& =2 \int_{0}^{x} d z z \int_{z}^{1+z-x} \frac{d u}{p^{2}(u)} .
\end{aligned}
$$

By inspecting the region of integration, we see that the last integral is equal to

$$
\int_{0}^{m} \frac{u^{2} d u}{p^{2}(u)}
$$

(where $m=\min (x, 1-x)$ ), plus an integral which is always finite. This plus (7.2) proves the lemma.

Lemma 7.2. If $S$ is a Borel set of capacity zero then any measure $\mu$ supported in $S$ has infinite energy. 
Proof. Let $E_{n}=\left\{u_{\mu} \leqq n\right\} \cap S$. Then $C\left(E_{n}\right)=0$ and $u_{\mu}$ is bounded on $E_{n}$. By Theorem 2.5, $\mu\left(E_{n}\right)=0$. Hence $\mu\left(\left\{u_{\mu}<\infty\right\} \cap S\right)=0$. Therefore

$$
E(\mu)=\int_{S} u_{\mu} d \mu=\infty .
$$

Theorem 7.1. If (7.1) is finite, then any $f$ on $(0,1)$ satisfying a Lipschitz condition has finite $I(f)$. If (7.1) is infinite, then $I(f)<\infty$ only when $f=$ const a.e.

Proof. The first part is immediate.

Suppose (7.1) is infinite. We will show that $E(\mu)=\infty$ for any nonzero $\mu$ on $(0,1)$. This will prove the theorem. For if $f$ is not constant a.e. and $I(f)<\infty$, then $E\left(d f^{*}\right)=I\left(f^{*}\right) \leqq I(f)$ by Theorems 1.3 and 2.1, and $d f^{*}$ is a nonzero measure.

Let $\nu$ be any measure on $(0,1)$. Then

$$
E(\nu, d x)=\int_{0}^{1} u_{\nu}(x) d x=\int_{0}^{1} d \nu(y) \int_{0}^{1} d x K(x, y)=\infty,
$$

by Lemma 7.1. Therefore $u_{v}$ is unbounded. Since every nonzero measure has an unbounded potential, the capacity of every set must be zero. Lemma 7.2 completes the proof.

A version of this theorem for general $\Psi$ and $p$ has been given by Preston [7]. His theorem is for the circle group rather than $(0,1)$.

8. The cases $p(x)=x^{\alpha}, 0<\alpha \leq \frac{1}{2}$. We consider first $0<\alpha<\frac{1}{2}$. We abandon the heuristic argument of section 3 , for the situation is quite different from the preceding cases. In fact,

$$
P(x) \sim 1 /(1-2 \alpha) \text { as } x \rightarrow 0,
$$

$$
K(x, y) \sim 2 \int_{y-x}^{y} P \sim 2 x /(1-2 \alpha), x<y, y \rightarrow 0 .
$$

This $K$ is like the Newtonian potential of a plane charge between grounded plates. Let $\delta_{a}$ be the $\delta$-function concentrated at $a$. Its potential function is $K(a, x)$. Now (8.1) shows that $K(a, x)$ is close to the constant $2 a /(1-2 \alpha)$ for $a \leqq x \leqq b$, if $b$ is small. Thus $\delta_{a}$ serves as an approximate equilibrium measure. By Theorem 2.7,

$$
C[a, b] \sim(1-2 \alpha) / 2 a \text { as } b \rightarrow 0, a<b .
$$

Then the proof of Theorem 4.3 gives

$$
\sigma^{2}\left[x, \frac{1}{2}\right] \sim(1-2 \alpha) / 2 x \text { as } x \rightarrow 0
$$

But this is no stronger than what we would get if we set $I(f)=\int_{0}^{1} f^{2}$ !

By comparison, Theorem 1.2 gives 


$$
\sigma\left[x, \frac{1}{2}\right] \leqq \text { const } x^{\alpha-1},
$$

which is too large by a factor $x^{\alpha-\frac{1}{2}}$.

The boundary case $p(x)=x^{1 / 2}$ is curious in that we have not found approximate equilibrium measures for intervals $[a, b]$. Nevertheless, the $\sigma[a, b]$ problem is solved approximately by a multiple of $\delta_{a}$, even though the potential $K(a, x)$ is hardly like 1 . Since

$$
K(a, b) \leqq K(a, x) \leqq K(a, a), \quad a \leqq x \leqq b,
$$

Theorem 2.7 gives

$$
1 / K(a, a) \leqq C[a, b] \leqq 1 / K(a, b)
$$

By (3.2) and (3.4),

$$
\begin{aligned}
P(x) & \sim \log (1 / x) \\
K(x, x) & \sim 2 \int_{0}^{x} P(t) d t \sim 2 x \log (1 / x) \text { as } x \rightarrow 0
\end{aligned}
$$

Hence

(8.4)

$$
C\left[x, \frac{1}{2}\right] \geqq \operatorname{const}\left(x \log \frac{1}{x}\right)^{-1} .
$$

To reverse this inequality, we will use the subadditivity of capacity. Suppose $2^{-n} \leqq x<2^{-n+1}, n=2,3, \cdots$. Then

$$
C\left[x, \frac{1}{2}\right] \leqq \sum_{k=1}^{n-1} C\left[2^{-k-1}, 2^{-k}\right]
$$

By (3.4) and (8.3),

$$
\begin{gathered}
K(t, 2 t) \sim 2 \int_{t}^{2 t} \log (1 / u) d u \sim 2 t \log (1 / t) \text { as } t \rightarrow 0, \\
C[t, 2 t] \leqq \frac{\text { const }}{t \log (1 / t)}, \quad 0<t \leqq 1 / 4
\end{gathered}
$$

Hence

$$
C\left[x, \frac{1}{2}\right] \leqq \text { const } \sum_{k=1}^{n-1} \frac{2^{k+1}}{k+1} \leqq \text { const } 2^{n} / n \leqq \text { const }\left(x \log \frac{1}{x}\right)^{-1} .
$$

It follows that $\sigma\left[x, \frac{1}{2}\right]$ is like

$$
\left(x \log \frac{1}{x}\right)^{-1 / 2}
$$

The result of Theorem 1.2, 


$$
\sigma\left[x, \frac{1}{2}\right] \leqq \text { const } x^{-1 / 2},
$$

is again too large by a factor $\log ^{1 / 2}(1 / x)$.

\section{References}

1. L. Carleson, Selected problems on exceptional sets, Van Nostrand, 1967.

2. A. M. Garsia, On the smoothness of functions satisfying certain integral inequalities, Proceedings of a Symposium on Functional Analysis, Academic Press, 1970, 127-162.

3. A. M. GARsia, Continuity properties of Gaussian processes with a multidimensional time parameter, Proceedings of the 6th Berkeley Symposium, 1970, (to appear).

4. A. M. Garsia, E. Rodemich, \& H. Rumsey, JR., A real variable lemma and the continuity of paths of some Gaussian processes, Indiana Univ. Math. J. 20 (1970), 565-578.

5. F. John \& L. Nirenberg, On functions of bounded mean oscillation, Comm. Pure and Appl. Math. 14 (1961), 415-426.

6. C. Preston, A theory of capacity and its application to some convergence results, Advances in Math. 6 (1971), 78-106.

7. C. Preston, Banach spaces arising from some integral inequalities, Indiana Univ. Math. J. 20 (1970), 997-1015.

8. H. TAYLOR, Rearrangements of incidence tables, J. Combinatorial Theory, (to appear).

University of Southern California

Los Angeles

Date communicated: AprIL 5, 1971 